\title{
A Practical Tool to Generate Complex Energy System Configurations Based on the SYNTHSEP Methodology
}

\author{
A. Toffolo ${ }^{1}$, A. Lazzaretto ${ }^{2 *}$ \\ ${ }^{1}$ Division of Energy Science, Dept. of Engineering Sciences and Mathematics, \\ Luleå University of Technology, Luleå, Sweden \\ ${ }^{2}$ Dept. of Industrial Engineering, University of Padova, Padova, Italy \\ E-mail: ${ }^{1}$ andrea.toffolo@ltu.se, ${ }^{2}$ andrea.lazzaretto@unipd.it
}

Received 01 January 2019, Accepted 27 February 2019

\begin{abstract}
Traditional fossil fueled power plants are commonly based on steam Rankine cycle or Brayton Joule cycle. Using water or air as working fluid is obviously the most obvious choice for the wide availability of these substances in nature. However, the scarcity of natural energy sources and the strong need of reducing environmental impact have necessarily drawn the research to new energy systems configurations operating with other working fluids, which are able to recover lower temperature sources, such as Sun or industrial wasted heat. The variety of new working fluids (refrigerants or organic fluids) widens the choice to a variety of configurations that can be tailored to the specific source characteristics and boundary constraints. It is not always easy or even possible to conceive the best configuration for given specifications with the mere experience of a common designer. To design a new system configuration, the designer normally uses some "non-codified rules" deriving from his knowledge of basic thermodynamics and energy engineering. This paper aims instead at showing a practical tool that is based on a new methodology, named SYNTHSEP, to generate new energy system configurations. This methodology starts from the simple thermodynamic cycles operated by a given fluid made up of the four fundamental processes (compression, heating, expansion and cooling) and uses a rigorous set of codified rules to build the final system configuration. The paper presents the basics of the new methodology and how it has been implemented in a practical tool that simply requires the information about the elementary cycles and their shared processes as input data.
\end{abstract}

Keywords: Synthesis, system configuration, optimization, software tool.

\section{Introduction}

The synthesis of system configuration is the ultimate problem, or "the" problem, in the field of the optimization of energy conversion systems. No standard procedure to deal with this problem exists in the scientific literature. Most of the synthesis optimization problems are solved using a superstructure, prepared by the designer in advance, and a pruning strategy for the search process leading to the definition of the optimal configuration (recent examples are, e.g., [1-3]).

In this context, the authors have identified first, as issues of critical importance, the internal heat recovery and the heat transfer with the external hot sources and cold sinks, and have developed the HEATSEP method to optimize these thermal interactions for a given system configuration [4-6]. Another critical issue is the identification of the components that are strictly necessary to realize the basic design concept behind an energy conversion system. In fact, these components can be organized according to elementary thermodynamic cycles, e.g., the steam injection gas turbine (STIG) cycle can be seen as formed by an air/gas Brayton cycle and a water/steam Rankine cycle. The performance of these cycles can be studied separately, in order to understand better which cycles should be attributed potential performance improvements [7].
More recently, the concepts previously formulated to analyze and improve existing system configurations have been employed to develop the SYNTHSEP methodology. This methodology goes in the opposite direction, i.e. generates new system configurations with a bottom-up approach, starting from elementary thermodynamic cycles, assembling them and proposing the resulting system configuration to the evaluation of an optimization algorithm.

While all steps of the SYNTHSEP methodology are presented in detail in [8-10], this paper is limited to the presentation of a practical software tool, specifically tailored to handle elementary Rankine cycles, in which the user specifies the information that is necessary for the generation of the basic configuration, which is then automatically assembled by the tool itself.

The paper is structured as follows. Section 2 provides some highlights about the background, the aim and the procedures of SYNTHSEP methodology. These highlights are useful for the reader as they cover the fundamental elements of the methodology that are necessary to understand the operation of the tool. Section 3 offers a description of the tool and the steps that the user has to follow from the submission of the input data about cycle design parameters and shared processes to the different forms of output about the resulting basic configuration (a drawing of the topology of the basic configuration, the list 
of node thermodynamic properties, the representation of processes in the temperature-specific entropy diagram). In Section 4, three examples of basic configurations are given which are assembled starting from the same three elementary cycles but having different processes shared by the cycles, in order to show a sample of the variety of configurations that can be obtained with the SYNTHSEP methodology.

\section{The SYNTHSEP Methodology}

\subsection{Background}

The SYNTHSEP methodology borrows many ideas from the HEATSEP method [4-6], which was conceived to simplify the way in which the flowsheet is analyzed in the design parameter optimization of a given energy conversion system. In the HEATSEP method, the focus of the designer is shifted to the set of the components that perform the fundamental processes in the flowsheet, the so-called "basic configuration" of the system. On the other hand, the heat transfer devices are removed and replaced with "thermal cuts" between the components belonging to the basic configuration, across which the temperature/enthalpy of the material flows is allowed to vary. All the heat transfers that are required to perform these temperature/enthalpy variations are assumed to occur inside a "black box" of unknown configuration, the definition of which is left to a subsequent step of the design process. A closer look at the basic configuration of energy conversion systems reveals that its components and the way in which they are organized simply reflect the essential design concept behind the system. This essential design concept is always expressed by a set of elementary thermodynamic cycles sharing some of their fundamental processes (compression, heating, expansion, cooling).

\subsection{Background}

The observations of the HEATSEP method on the basic configurations of existing energy conversion systems, i.e. the identification of elementary thermodynamic cycles as the units forming the basic configuration, can be exploited in reverse to artificially generate basic configurations starting from elementary thermodynamic cycles as candidate solutions to synthesis/design optimization problems.

The SYNTHSEP methodology defines a framework in which elementary thermodynamic cycles can be assembled into a basic configuration through a rigorous set of logical rules applied to the information about design parameters of the cycles and the processes that are shared by the cycles.

The aim of the SYNTHSEP methodology is to use this assembling framework as an innovative approach to represent and explore the search space of synthesis/design optimization problems. The traditional top-down approach of having experts define a superstructure to be pruned during the search process is replaced by a bottom-up approach starting from the unit "bricks" of the candidate solutions, i.e. the elementary thermodynamic cycles. The set of rules defined in the SYNTHSEP methodology for assembling the elementary thermodynamic cycles into a basic configuration can be seen as a way to define an "open" superstructure that can be handled by an artificial intelligence and is no longer limited by the expertise of the designer.

\subsection{Definitions and Procedures}

The goal of this section is to summarize the fundamental definitions and procedures of the SYNTHSEP methodology, in order to make the user able to understand the essentials about the operation of the tool. It is obvious that this summary has to start from the elementary thermodynamic cycles, the unit "bricks" from which the basic configuration is assembled.

Elementary Rankine cycles operate across the phase change zone and perform the following processes in this order (see Figure 1): compression (A), heating (B), expansion (C) and cooling (D). All their design parameters are completely defined by the knowledge of the thermodynamic states (characterized by pressure and temperature/enthalpy) at the beginning of the compression and at the beginning of the expansion. In fact, the isentropic efficiencies of the compression and the expansions, which are considered as constants here, could be defined as a function of the design parameters themselves, such as the pressure changes.

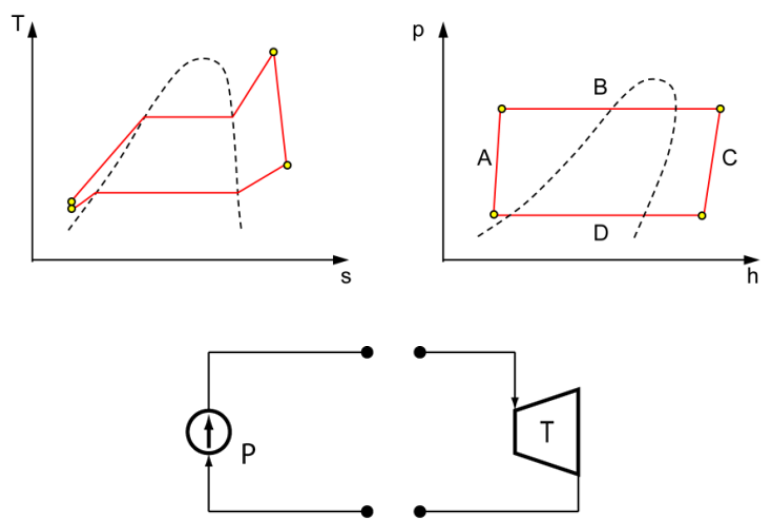

Figure 1. An elementary Rankine cycle represented in the temperature-specific entropy diagram (top-left), in the pressure-specific enthalpy diagram (top-right) and according to the HEATSEP method (bottom) with thermal cuts indicating the heating and cooling processes.

The elementary cycles are aggregated into a basic configuration by a list of shared processes (of course the list may be empty, and in this case the basic configuration is made of a set of separate cycles). Each shared process in the list can be identified by the type (A, B, C or D) and a set of $n$ Boolean flags (where $n$ is the number of elementary cycles forming the basic configuration) to mark the cycles involved. For instance, in Figure 2 the two cycles forming the basic configuration share the compression and heating processes, so the two shared processes in the list are A:[11] and $\mathrm{B}:[11]$.

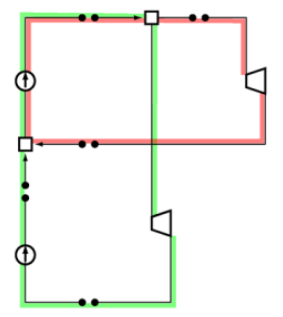

$A:[1$ 1] compression

$B:[11]$ heating expansion cooling

Figure 2. An example of basic configuration made of two elementary thermodynamic cycles sharing the compression and heating processes (Figure is in color in the on-line version of the paper). 
The possible combinations of shared processes increase with the number of cycles, so the list of shared processes (and the corresponding topology of the basic configuration) may become more complex. The following list of shared processes is made for a basic configuration made of four elementary cycles, in which cycles 1 and 3 share a compression process, cycles 2 and 4 share another compression process, cycles 1, 2 and 4 share an expansion process and cycles 3 and 4 share a cooling process:

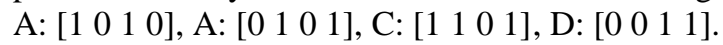

Since the elementary cycles may have their processes shared partially or totally with other cycles, in the SYNTHSEP framework the following definition of "shared process" is given in [9].

A process shared by two or more elementary cycles is defined as a sequence of process segments. For shared compression/expansion processes, the segments connect the ordered list of the pressure levels of the cycles involved in the shared process itself. For shared heating/cooling processes, the segments connect the ordered list of the temperature/enthalpy levels of the cycles involved in the shared process itself.

In this way, the shared process encompasses the whole range of pressures or of temperatures/enthalpies of the cycles involved, but the mass flow rates of the cycles participate only in the segments of the shared process that are within the range determined by their minimum and maximum pressures or minimum and maximum temperatures/enthalpies. As a consequence, mixers and splitters must be introduced to delimit the segments of the shared process wherever mass flow rates are to be merged or split, respectively.

Temperatures are allowed to vary between the segments along a shared compression/expansion process to evaluate opportunities for improving the internal and/or external heat transfer (see, e.g., systems with reheating and intercooling). However, an incorrect interaction of mixers and splitters with the thermal cuts making these additional heating and cooling processes possible may cause a mass flow rate to undergo two thermal processes of opposite sign one immediately after the other (see the top diagram in Figure 3). Some specific rules of the SYNTHSEP methodology are identified to avoid this problem and to correctly assemble the elementary cycles in the shared compression/expansion processes of the basic configuration.

When a cycle joins a shared compression or expansion process, the temperature at the end of the segment before the mixer is always brought at the temperature of the incoming mass flow rate, and the thermal cut for this additional heating or cooling process has known initial and final temperatures (see the bottom diagram in Figure 3). Conversely, when a cycle abandons a shared compression or expansion process, first its mass flow rate is made to leave the shared process, then, after the splitter, the mass flow rate continuing along the shared process is allowed to vary its temperature before the next compression or expansion segment (see the bottom diagram in Figure 3). In the second case, the final temperature of the thermal cut for this additional heating or cooling process cannot be found among those supplied with cycle data, so additional data (temperatures) have to be provided to fix the additional degrees of freedom created at the beginning of the segments compression or expansion after a splitter along shared processes (see Figure 3).

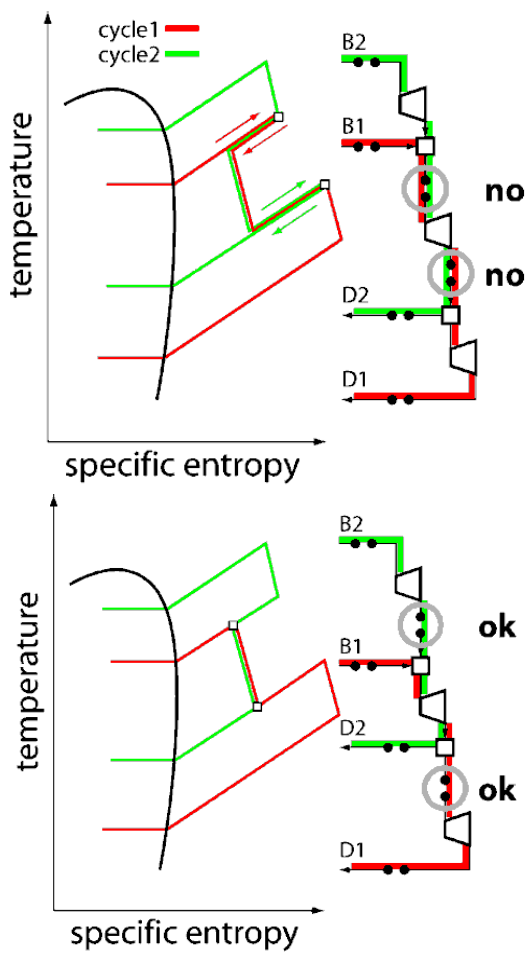

Figure 3. Incorrect (top) and correct (bottom) positions for the insertion of the additional thermal cuts in a basic configuration made of two cycles sharing the expansion process [8] (Figure is in color in the on-line version of the paper).

The minimum and maximum pressures and temperatures of the cycles, the list of the shared processes and the additional temperatures fixing the additional degrees of freedom after the splitters along shared compression or expansion processes completely define the topology and the design parameters of a basic configuration. However, some pieces of information contained in this set of data may be conflicting. For instance, if two cycles with different evaporation pressure levels are supposed to share the heating process, only one of the two levels can be selected to make it happen.

Thus, the procedure followed by the SYNTHSEP methodology to assemble the elementary cycles into a basic configuration necessarily comprises a reconciliation step that eliminates the discrepancies contained in the input data according to a rigorous set of logical rules. In general terms, these rules contain instructions about which information has the precedence, which parameters are used, which are overridden by others, or which are simply ignored.

The ultimate goal of the SYNTHSEP methodology is to have the procedure to assemble the basic configuration starting from a set of input data completely automated, while new input data are continuously proposed by a hybrid evolutionary optimization algorithm as in [8-10].

\section{A description of the Tool}

The tool to assemble a basic configuration starting from elementary thermodynamic cycles is written in MATLAB programming language and is compiled into an executable file (.exe) that can be copied to the computer of the user. In case the user does not have access to MATLAB, the executable can be run anyway with the help of MATLAB Runtime shared libraries, which must be installed on the computer of the user. 


\subsection{Submission of Input Data}

When the tool is executed, a window appears on the screen where the user is welcomed and given some basic instructions about the tool, among which the limitations on the pressure and temperature ranges for calculating the properties of the operating fluid (at the moment the tool works only with water/steam between 0.01 and 200 bar and between 0 and $500^{\circ} \mathrm{C}$, and the properties are calculated with the CoolProp library. Pressure and temperature ranges are of course specific for the considered operating fluid). In the same window, the user is also invited to select the number of elementary thermodynamic cycles that will form the basic configuration (from 2 to 6 ).

When the selection is confirmed, a new window ("Input data submission") appears in which the information about the elementary thermodynamic cycles and their shared processes is to be provided. The window is divided into two panels (Figure 4). In the left panel the user is invited to fill in for each cycle the data about the two thermodynamic states that univocally define the four processes, i.e. the state at the beginning of the compression (minimum pressure and temperature of the cycle, in the liquid phase zone) and the state at the beginning of the expansion (maximum pressure and temperature of the cycle, in the vapor phase zone). The values of these quantities have to be specified for each cycle in a row of four fields accepting numerical input. The right panel of the window is where the shared processes among the elementary cycles of the basic configuration are defined. Each of the 12 rows of interface controls in the panel represents a potential shared process and is made of one pop-up menu and as many checkboxes as the number of elementary cycles forming the basic configuration (12 potential shared processes is a limitation due to window size only, but of course, e.g., basic configurations made of two cycles cannot support more than 3 shared processes, see [10]). A shared process is defined by the type (A, B, C or $\mathrm{D}$ ) of the process, to be selected from the pop-up menu, and by the cycles that are involved in it, to be selected by ticking the corresponding checkboxes. Rows with no inputs (i.e. no letter in the popup menu and no checkboxes ticked) are simply ignored. When the user has inserted all the relevant information in both panels, the "Check and submit" button at the bottom of the window is to be clicked. At this point the input data are checked to prevent mistakes that may affect the subsequent steps of the procedure, and if mistakes are present the corresponding interface controls are highlighted in red and the user is asked to modify those inputs. Here are examples of mistakes that can be detected:

- Non-numeric input in the fields of cycle data;

- Cycle data are outside the ranges in which operating fluid properties can be calculated;

- The maximum pressure of the cycle is lower than the minimum one, or vice versa;

- The provided temperature, for the provided pressure, defines a state out of the expected phase zone (in this case the provided temperature is not only highlighted in red but replaced with the indication of the expected range);

- A shared process has zero or one checkboxes ticked (i.e. involves no cycles or just one cycle);

- In a shared process some checkboxes are ticked but the process type is not selected.

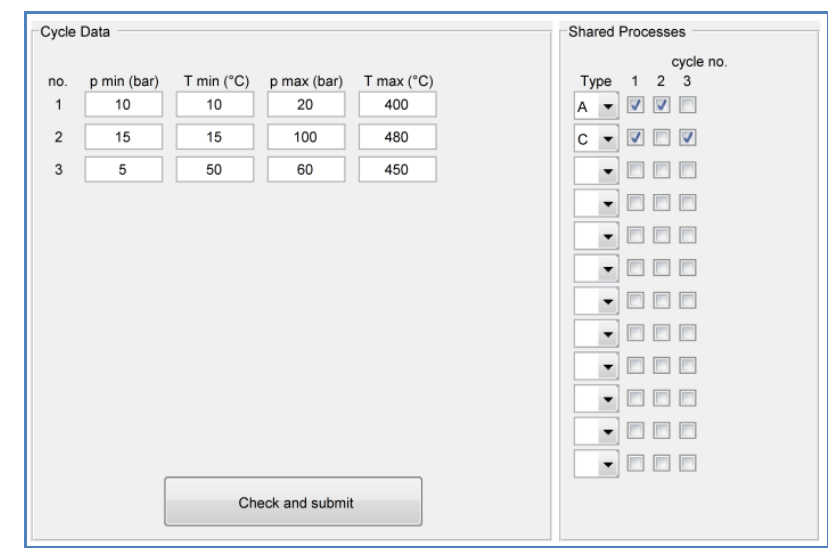

Figure 4. "Input data submission” window.

\subsection{Assembling the Topology of the Basic Configuration}

When the information provided in the "Input data submission" window is free of mistakes, the window is automatically closed and the tool proceeds to assemble the elementary cycles into the topology of the basic configuration according to the data about the thermodynamic states of the cycles and the list of the shared processes.

This step of the procedure, which is the core of the tool, is performed according to a rigorous set of logical rules to reconcile the possible conflicts between the information about cycle data and the information about the shared processes. Using these rules, the pressure levels of the cycles are first reviewed according to the shared heating and cooling processes to be ordered into those of the basic configuration. Then, every piece of the topology of the basic configuration is determined, starting from the segments of the shared compression and expansion processes, in which mixers and splitters and additional thermal cuts have to be inserted. Non-shared compression and expansion process are determined next, and finally heating and cooling processes (shared and non-shared) just have to be placed to connect the nodes of the topology that have already been created in the calculation about compressions and expansions.

When the calculation about the topology is accomplished, two windows appear on the screen, the "Topology of the basic configuration" window and the "Basic configuration data" window (Figure 5).

In the "Topology of the basic configuration" window a rough sketch of the topology is drawn. Processes are represented by arrows connecting numbered nodes, which represent the thermodynamic states at the ends of the processes. Vertical lines with upward arrows on the left of the window are compression processes and vertical lines with downward arrows on the right of the window are expansion processes. Horizontal lines with arrows pointing right in the top of the window are heating processes and horizontal lines with arrows pointing left in the bottom of the window are cooling processes. Mixers and splitters can be identified by small squares plotted on top of the corresponding node of the topology. In this representation the elementary thermodynamic cycles can be easily recognized as rectangles, and the segments of the shared processes in which their mass flow rates are merged are seen as the segments in which the sides of the rectangles are overlapping. For instance, in Figure 5 cycle 1 can be identified as the rectangle having nodes 7, 8, 9 and 14 as 
vertices, and cycle 2 as the rectangle having nodes $1,4,11$ and 12 as vertices. Cycle 1 and 2 share the expansion process (see the "C: [110]" shared process in the bottom panel of the "Basic configuration data" window) and their mass flow rates are merged between the mixer (node 11) and the splitter (node 12), i.e. along the common segment between the right sides of the two rectangles.

In the "Basic configuration data" window the information about cycle data and shared process is shown again in two panels after the assembling of the elementary cycles into the basic configuration. In the bottom panel the information about the shared processes is presented under the form of lists for each of the four process categories (A, $\mathrm{B}, \mathrm{C}$ and D). The lists are made of vectors of binary values, and the binary values in each vector mark the cycles that are involved in the shared process represented by that vector.

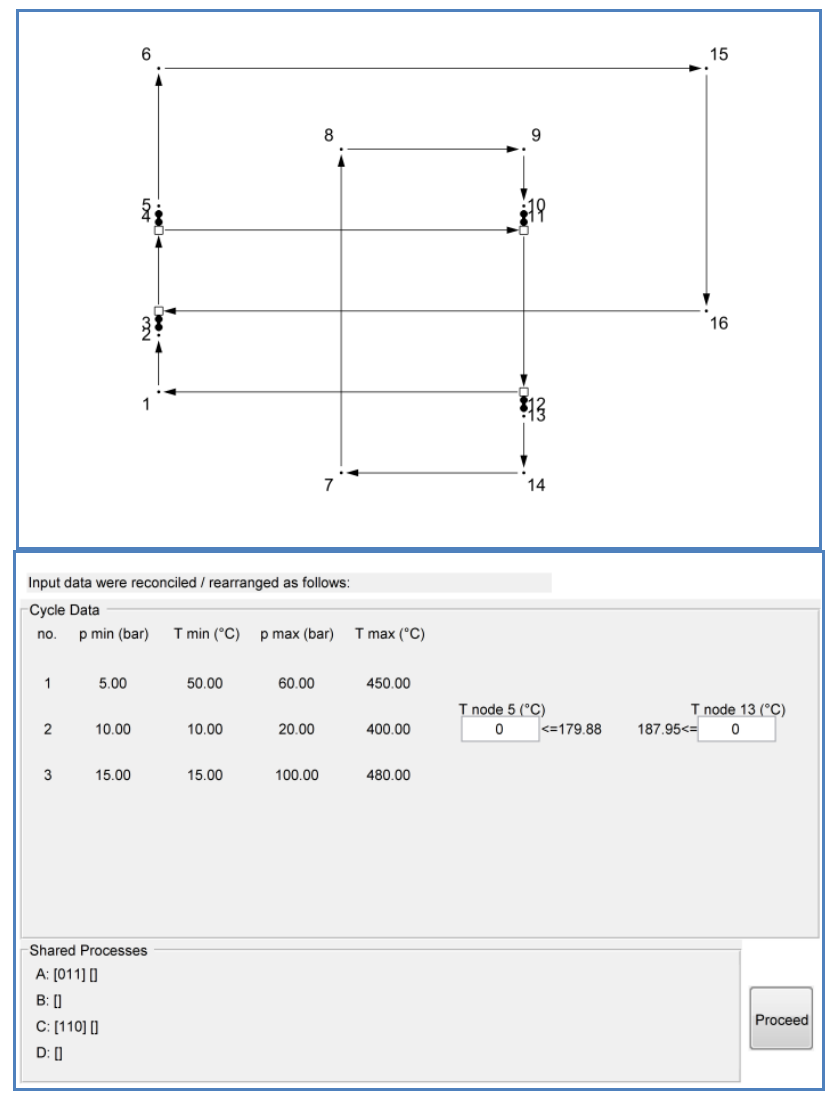

Figure 5. "Topology of the basic configuration" window (top) and "Basic configuration data" window (bottom).

The upper panel reports the minimum and maximum pressures and temperatures of the cycles after the reconciliation with the information about the shared processes. Please note that in the upper panel about cycle data the order of the cycles, as given by the user in the "Input data submission" window, may have been rearranged (the order of the cycles in the binary value vectors representing the shared processes is rearranged accordingly in this case, compare Figures 4 and 5). Note also that also some temperatures in the cycle data are changed if the associated pressure has been changed. In fact, temperatures are converted by the procedure into real numbers in the range between 0 and 1 (e.g., in the vapor phase zone, 0 corresponds to the saturation temperature at that pressure level and 1 to the higher end of the considered temperature range for the operating fluid, in this case
$500{ }^{\circ} \mathrm{C}$ ), so that there is no risk that a node at the beginning of a compression or expansion process ends up in the phase change zone when its pressure has been changed due to the reconciliation of the information about shared heating or cooling processes.

Moreover, some numerical fields may be present in the upper panel to accept user input about the temperatures corresponding to the additional degrees of freedom that are created whenever the mass flow rate of one cycle leaves a shared compression or expansion process from a splitter (see Section 2.3). Each numerical field is titled with the number of the node it refers to (the node along the shared compression or process after the splitter) and an indication is given about the expected range of the temperature to be provided, so that the node belongs to the correct phase zone (in Figure 5 the temperature of node 5 has to be lower than $179.88^{\circ} \mathrm{C}$ and that of node 13 must be higher than $\left.187.95^{\circ} \mathrm{C}\right)$. User input is checked when the "Proceed" button is clicked before passing to the final step of the procedure that provides the output of the basic configuration.

\subsection{Output Data}

When the last possible user input step is completed, the tool provides a series of data about the resulting basic configuration and stops running. Two new windows appear on the screen, the "T-s diagram" window and the "Node data" window (Figure 6). The "T-s diagram" window shows the thermodynamic processes of the basic configuration in the temperature-specific entropy diagram.
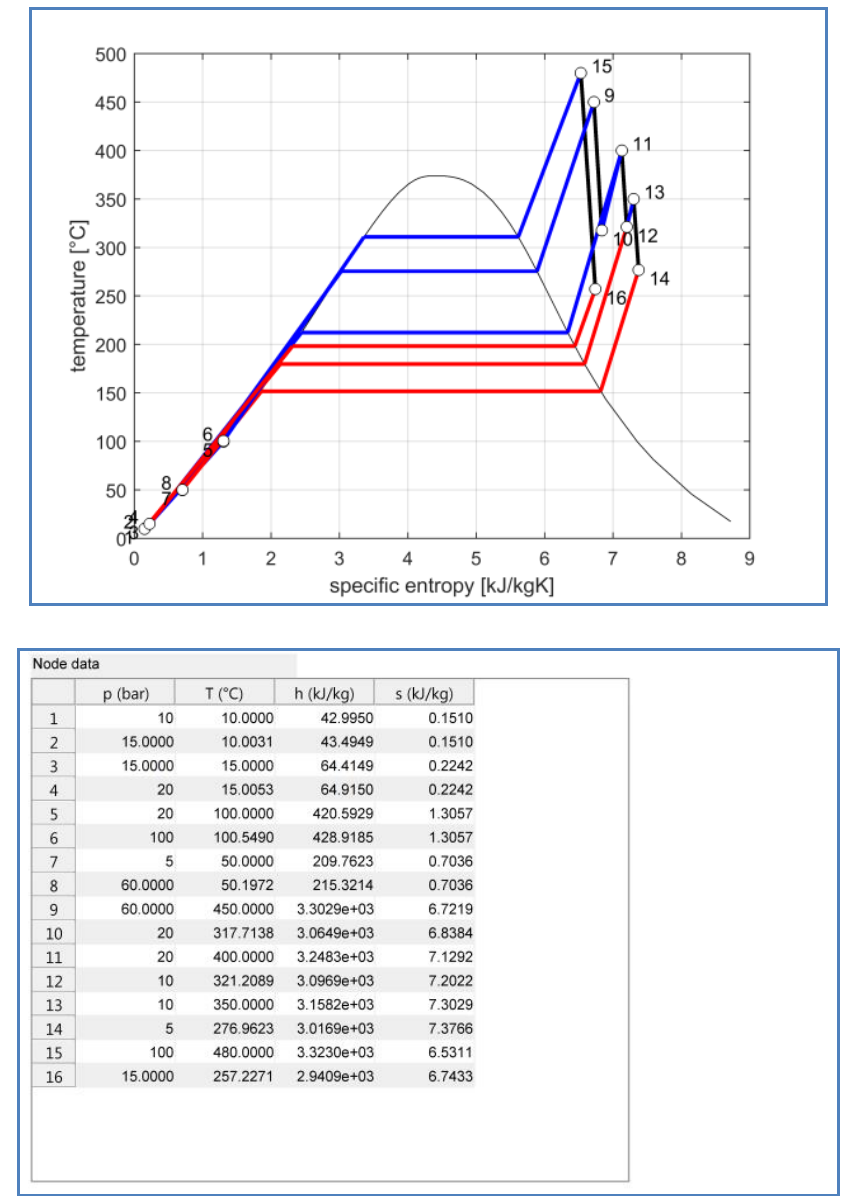

Figure 6. "T-s diagram" window (top) and "Node data" window (bottom) (Figure is in color in the on-line version of the paper). 
- Heating processes and the additional thermal cuts across which the temperature rises along shared compression and expansion processes are shown as blue lines (they all are actually cold thermal streams from the Pinch Analysis [11-13] point of view, as they require heat to make the temperature increase);

- Cooling processes and the additional thermal cuts across which the temperature drops along shared compression and expansion processes are shown as red lines (they all are actually hot thermal streams from the Pinch Analysis point of view, as they release heat to make the temperature decrease);

- Black lines represent compression and expansion processes.

The small circles at the ends of the processes are numbered to show the correspondence between the thermodynamic states in the T-s diagram and the nodes in the "Topology of the basic configuration" window.

Finally, the "Node data" window shows a table including pressure, temperature, specific enthalpy and specific entropy at each node of the topology.

\section{Examples of Basic Configurations Formed by Three Elementary Cycles}

In this section three examples of basic configurations made of three elementary cycles are proposed and their features discussed. These examples should be sufficient to show the wide variety of basic configuration topologies that can be obtained just by varying the list of the shared processes among the same three elementary thermodynamic cycles (in fact, cycle data provided in the "Input data submission" window are the same in all three examples, see Figure 7).

It is assumed that these basic configurations are assembled for steam cycles that are to be thermally matched to some external processes that can provide heat to the steam cycle and can recover the heat released by the steam cycle, while the temperature gap between the external hot sources and cold sinks is exploited by the steam cycle to generate as much power as possible.

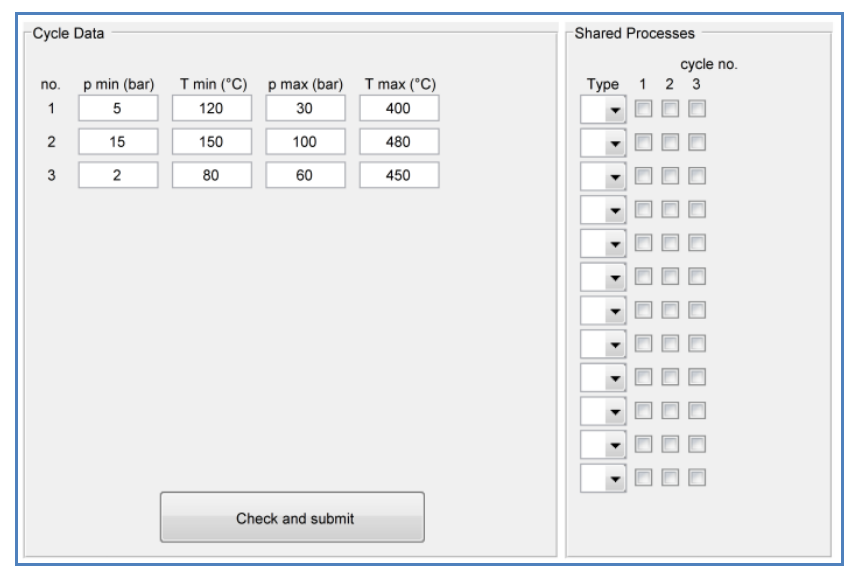

Figure 7. "Input data submission" window with the design parameters of the three elementary Rankine cycles that are used in all the three examples of basic configurations (please note that the order and the parameters of the cycles may be altered by the reconciliation step in Figures 8 to 10).

\subsection{Example 1}

In this example the list of shared processes to be inserted in the "Input data submission" window is: A:[111], $\mathrm{C}:[111]$. Accordingly, the resulting basic configuration is chosen to have all the three cycles share the compression and expansion processes (Figure 8). The evaporation and condensation pressure levels of the cycles are not altered by the shared processes, so there are three evaporation and three condensation pressure levels. There are four additional degrees of freedom: the two temperatures to be specified after the two splitters along the shared compression and the two after the two splitters along the shared expansion.

The thermal matching between this basic configuration and the external hot sources is highly flexible for two reasons:

- The three available evaporation pressure levels offer a high chance of minimizing the exergy destruction associated with the heat transfer both in case of a single source providing sensible heat and in case of multiple sources at variable and constant temperature.

- Heating and cooling processes before the mixers and after the splitters along the shared expansion provide an opportunity to adjust the slope of the temperature profiles in the temperature range of superheating streams and, less significantly perhaps, in that of the preheating streams.

The three condensation pressure levels also ensure high flexibility in the thermal matching with the external cold sinks, such as industrial processes in the medium-low and low temperature ranges. The majority of the heat released from the cooling processes of the configuration comes of course from condensations at constant temperature, so increasing the number of condensation levels is fundamental to better adapt the heat release to the temperature profiles of the cold sinks.

\subsection{Example 2}

In this example the list of shared processes to be inserted in the "Input data submission" window is: B:[111], $\mathrm{C}:[111]$. Accordingly, the resulting basic configuration is chosen to have all the three cycles share the heating and expansion processes. In this configuration (Figure 9) the three evaporation pressure levels are necessarily merged into one (the one at highest pressure) to fulfil the requirement about the shared heating process, whereas there are still three separate condensation pressure levels. There are two additional degrees of freedom along the shared expansion process, one after each splitter.

Due to the single evaporation pressure level, the flexibility in the thermal matching between this basic configuration and the external hot sources is severely limited in the case of a single hot source at variable or constant temperature, and only a lucky combination of sources at variable and constant temperature could minimize the gap between the hot (external) and cold (basic configuration) temperature profiles. A minor contribution to adjust the slope of the temperature profile of the (single) superheating stream with reheating processes could come from the two additional degrees of freedom after the splitters along the shared expansion process. 


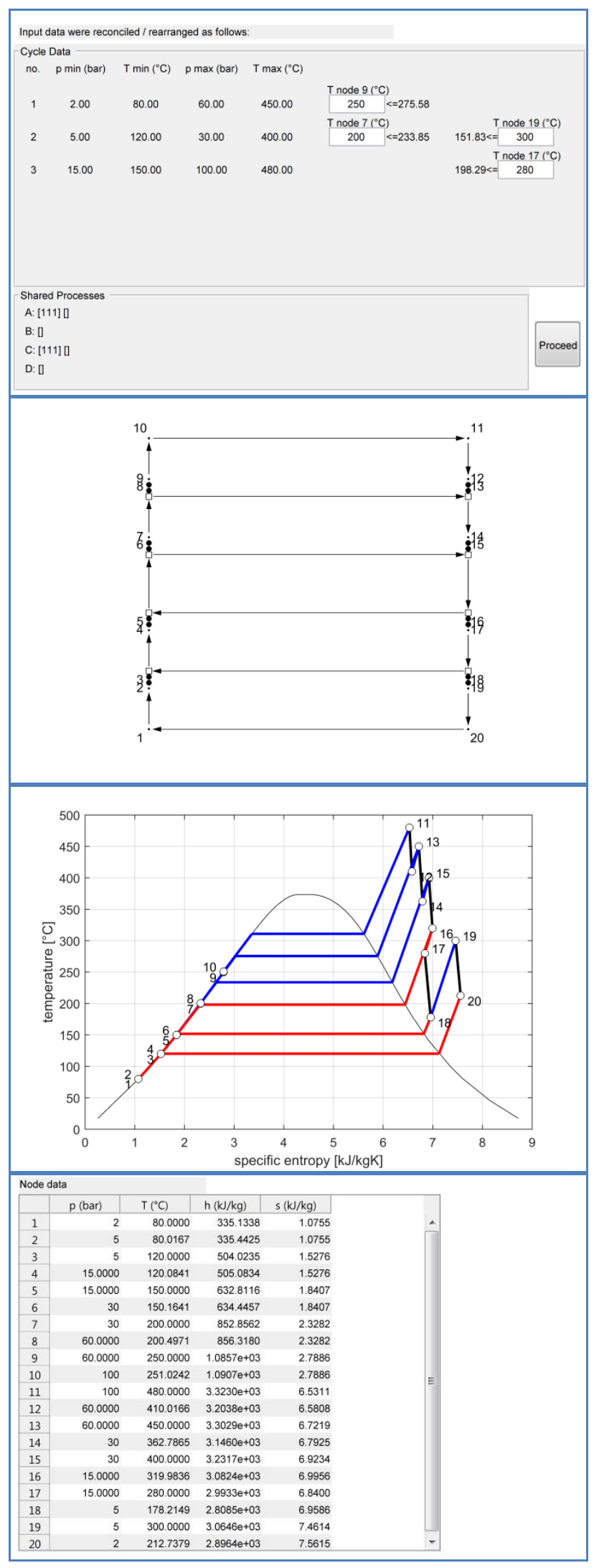

Figure 8. Tool windows about the basic configuration of example 1 (Figure is in color in the on-line version of the paper).

The flexibility in the thermal matching with the external cold sinks is again optimal thanks to the maximum number (three) of condensation pressure levels available.

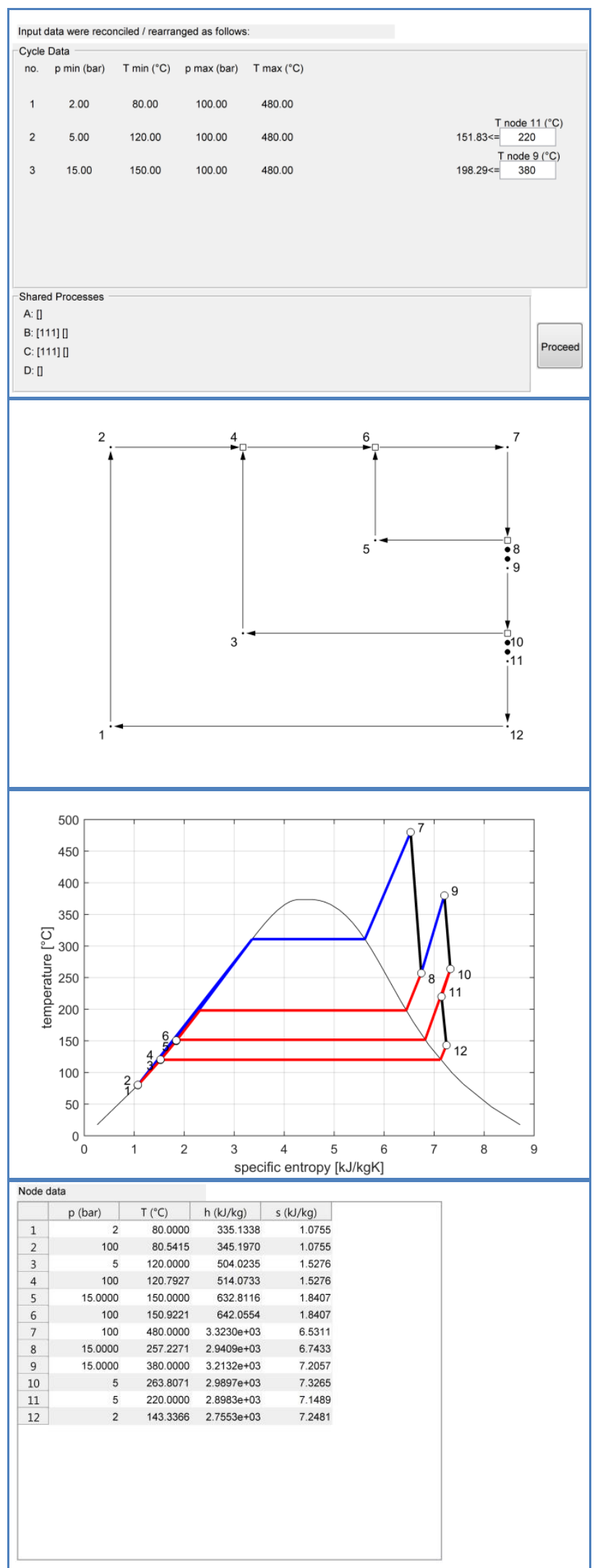

Figure 9. Tool windows about the basic configuration of example 2 (Figure is in color in the on-line version of the paper).

\subsection{Example 3}

In this example the list of shared processes to be inserted in the "Input data submission" window is: A:[101], $\mathrm{C}:[110], \mathrm{D}:[111]$. Accordingly, the resulting basic configuration is chosen to have three shared processes 
(Figure 10): a shared compression involving cycles 1 and 2 (cycle numbering here refers to Figure 10, that is after the reconciliation step of the assembling procedure), a shared expansion involving cycles 1 and 3 and a shared cooling process involving all three cycles. The cooling process shared by the three cycles requires a common condensation pressure level (the one at lowest pressure), whereas the evaporation pressure levels are not modified by the reconciliation step of the procedure. The only additional degree of freedom is the temperature after the splitter along the compression shared by cycles 1 and 2 .

The three evaporation pressure levels ensure a good flexibility in the thermal matching with the external hot sources, although in this configuration there are no additional degrees of freedom along the expansion shared by cycles 1 and 3 . So the only opportunity to adjust the slope of the temperature profiles of the superheating streams is to change the temperature of node 9 , i.e. the maximum temperature of cycle 1 in Figure 10.

The single condensation pressure level is certainly not ideal for a thermal matching with several external cold sinks at constant and variable temperature. Thus, this basic configuration is fit to maximize the generation of power by rejecting all the heat at the lowest possible temperature, indeed determined by the lowest condensation level of the three cycles.

\section{Conclusions}

This paper presented practical aspects about the assembling of elementary thermodynamic cycles into a basic configuration according to the SYNTHSEP methodology. The goal is to demonstrate the generality of the assembling procedure and the user-friendliness of an automatic tool implementing this procedure, in spite of the quite complex rules contained in it.

Three examples of basic configurations were built deriving from different choices of the processes shared by the elementary thermodynamic cycles, in order to show that:

- The user is easily guided by the tool in supplying simple pieces of information, namely the minimum and maximum pressures and temperatures of each cycle, the list of the shared processes and, in case, additional node temperatures specifically requested by the tool;

- The general and rigorous rules of the assembling procedure are able to generate basic configurations that would be hardly conceived in a short time, even by an expert designer, because of the complexity of the internal and external thermal interactions;

- The generated basic configurations not only exploit profitably the internal heat recovery, but also are able to adapt to different types of external hot sources and cold sinks.

The goal of this automated assembling procedure of the SYNTHSEP methodology is indeed to propose basic configurations as candidate solutions to an optimization algorithm in order to find the optimal thermal interactions, according to a given objective function, taking into account the heat transfer with the hot source, the heat transfer with the cold sinks and the internal heat recovery.

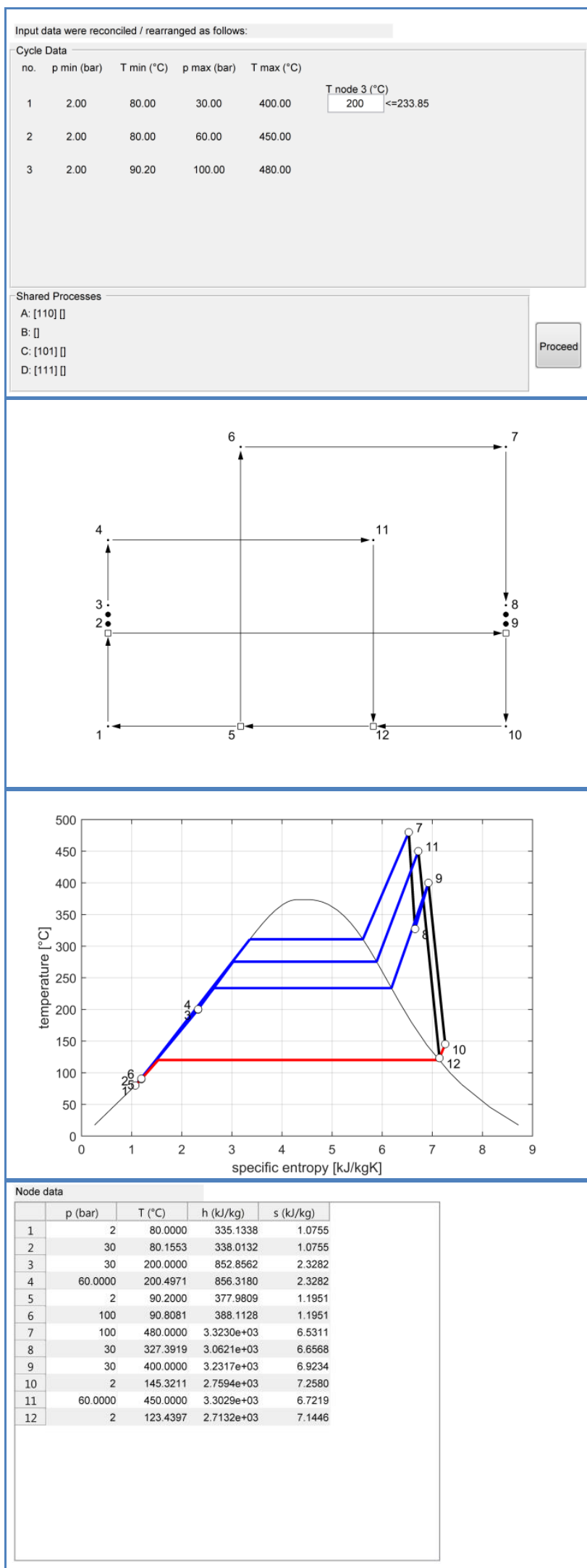

Figure 10. Tool windows about the basic configuration of example 3 (Figure is in color in the on-line version of the paper).

\section{References}

[1] M. O. Bertran, R. Frauzem, A. S. Sanchez-Arcilla, L. Zhang, J. M. Woodley, R. Gani, "A generic methodology for processing route synthesis and design based on superstructure optimization," Computers \& Chemical Engineering, 106, 892-910, 2017. 
[2] C. Cui, X. Li, H. Sui, J. Sun, "Optimization of coalbased methanol distillation scheme using process superstructure method to maximize energy efficiency," Energy, 119, 110-120, 2017.

[3] S. Kwon, W. Won, J. Kim, “A superstructure model of an isolated power supply system using renewable energy: Development and application to Jeju Island, Korea," Renewable Energy, 97, 177-188, 2016.

[4] A. Lazzaretto, F. Segato, "Thermodynamic optimization of the HAT cycle plant structure - Part I: Optimization of the "Basic Plant Configuration"," ASME Journal of Engineering for Gas Turbines and Power, 123, 1-7, 2001.

[5] A. Lazzaretto, F. Segato, "Thermodynamic optimization of the HAT cycle plant structure - Part II: Structure of the heat exchanger network," ASME Journal of Engineering for Gas Turbines and Power, 123, 8-16, 2001.

[6] A. Lazzaretto, A. Toffolo, "A method to separate the problem of heat transfer interactions in the synthesis of thermal systems," Energy, 33, 163-170, 2008.

[7] M. Morandin, A. Toffolo, A. Lazzaretto, "Superimposition of elementary thermodynamic cycles and separation of the heat transfer section in energy systems analysis," ASME Journal of Energy Resources Technology, 135, 021602, 2013

[8] A. Toffolo, "A synthesis/design optimization algorithm for Rankine cycle based energy systems," Energy, 66, 115-127, 2014.

[9] A. Toffolo, S. Rech, A. Lazzaretto," Combination of elementary processes to form a general energy system configuration," in IMECE 2017: Proceedings of the ASME International Mechanical Engineering Congress and Exposition: IMECE2017-71653.

[10] A. Lazzaretto, G. Manente, A. Toffolo, "SYNTHSEP: A general methodology for the synthesis of energy system configurations beyond superstructures," Energy, 147, 924-949, 2018.

[11] B. Linnhoff, "Pinch technology for the synthesis of optimal heat and power systems," ASME Journal of Energy Resources Technology, 111, 137-147, 1989.

[12] B. Linnhoff (ed.), A user guide on process integration for the efficient use of energy. Rugby, UK: Institution of Chemical Engineers, 1982.

[13] I. C. Kemp, Pinch analysis and process integration: a user guide on process integration for the efficient use of energy. Oxford, UK: Butterworth-Heinemann, 2011. 